\title{
Abnormal Neural Oscillatory Activity to Speech Sounds in Schizophrenia: A Magnetoencephalography Study
}

\author{
Shogo Hirano, Yoji Hirano, Toshihiko Maekawa, Choji Obayashi, Naoya Oribe, Toshihide Kuroki, Shigenobu Kanba, \\ and Toshiaki Onitsuka \\ Department of Neuropsychiatry, Graduate School of Medical Sciences, Kyushu University, Fukuoka 812-8582, Japan
}

\begin{abstract}
Schizophrenia impairs many cognitive functions, and abnormalities in language processing have been proposed as one of the bases for this disorder. Previously, it was reported that different magnetoencephalography (MEG) patterns of the evoked oscillatory activity (eOA) of $20-45 \mathrm{~Hz}$ to speech and nonspeech sounds were evidence of a fast mechanism for the representation and identification of speech sounds in humans. The current study tested the hypothesis that the schizophrenics would show abnormal neural oscillatory activity, as measured by $\mathrm{eOA}$, to speech and nonspeech sounds. Twenty patients and 23 control subjects participated in this study. MEG responses to speech and nonspeech sounds were recorded and $\mathrm{eOA}$ power and phase locking at $20-45 \mathrm{~Hz}$ were analyzed. Patients showed significantly delayed peak latencies of the e $\mathrm{OA}$ power and phase locking to speech sounds in the left hemisphere and to nonspeech sounds in the right hemisphere. Patients also showed a significantly reduced eOA power to speech sounds in the left hemisphere in $0-50 \mathrm{~ms}$ and a significantly larger eOA power to speech sounds in the left hemisphere in 100-150 ms. In addition, the analyses of the lateralization index revealed the pattern of hemispheric lateralization to be the opposite in patients. These results indicated that patients showed different characteristics of eOA compared with normal controls, probably related to deficits in a fast mechanism for identifying speech sounds. Moreover, the present study suggests that schizophrenia might be characterized by an opposite pattern of hemispheric lateralization in auditory evoked oscillations.
\end{abstract}

Key words: evoked oscillatory activity; schizophrenia; speech sounds; magnetoencephalography; lateralization; wavelet transform

\section{Introduction}

Speech sound recognition may be qualitatively different from other recognition systems in terms of the underlying neurobiological structure and developmental trajectory. Belin et al. (2000) reported that human voices were perceived, at least in part, by a processing stream separate from that used in processing other nonvocal environmental sounds. From an evolutionary perspective, speech sound recognition represents a significant element of verbal decoding to solve adaptive problems critical for survival. Accordingly, the human brain must have a fast mechanism for representing and identifying speech sounds.

The neural oscillatory activity in high-frequency, in particular, in the gamma $(30-80 \mathrm{~Hz})$ and beta $(15-30 \mathrm{~Hz})$ bands, is supposed to play a critical role in feature binding and object representation (Uhlhaas and Singer, 2006). The functional roles of the evoked and induced oscillatory activity have been specifically investigated in humans (Uhlhaas and Singer, 2006). The

Received June 14, 2007; revised March 21, 2008; accepted March 21, 2008.

This work was supported in part by a grant from Research Group For Schizophrenia, Japan (T.0.), Grants-in-Aid for Scientific Research C17591218 (T.0.) and B19390306 (S.K.) from the Ministry of Education, Culture, Sports, Science, and Technology, Japan, and Grant H18 kokoro-ippan-012 from Ministry of Health, Labor and Welfare, Japan (S.K.). Our appreciation is expressed to Drs. Takefumi Ueno, Takehito Hayami, and Seiichi Sakatani for clarification regarding methodology. We gratefully acknowledge the technical support of Yuko Somehara.

Correspondence should be addressed to Dr. Toshiaki Onitsuka, Department of Neuropsychiatry, Graduate Schoo of Medical Sciences, Kyushu University, 3-1-1, Maidashi, Higashiku, Fukuoka 812-8582, Japan. E-mail: toshiaki@npsych.med.kyushu-u.ac.jp.

D0I:10.1523/JNEUROSCI.5031-07.2008

Copyright $\odot 2008$ Society for Neuroscience $\quad$ 0270-6474/08/284897-07\$15.00/0 evoked oscillatory activity (eOA) is phase locked to the onset of stimuli. Herrmann et al. (2004) suggested that the eOA occurring within $150 \mathrm{~ms}$ after stimulation reflected the rapid matching of bottom-up signals with memory contents.

In schizophrenia, abnormal eOA has been repeatedly reported in auditory and visual perception processes. In the auditory gamma band eOA, Kwon et al. (1999) reported reduced $40 \mathrm{~Hz}$ steady-state eOA in schizophrenia. Hong et al. (2004) showed reduced steady-state $\mathrm{eOA}$ at $40 \mathrm{~Hz}$ in first-degree relatives with schizophrenia spectrum personality symptoms. For visual perception, Spencer et al. $(2003,2004)$ demonstrated that visual gestalt stimuli lead to abnormal eOA at $\sim 40 \mathrm{~Hz}$ over the occipital lobe in schizophrenia, and that this abnormal eOA was associated with visual hallucinations. In the beta band, Uhlhaas et al. (2006) reported that the deficits in Gestalt perception in schizophrenia were associated with reduced phase-synchrony of eOA.

Magnetoencephalography (MEG) offers millisecond resolution to detect neuronal activity to auditory stimuli. Palva et al. (2002) reported different MEG patterns of the eOA within 100 ms after stimulation to speech and nonspeech sounds in healthy subjects. They interpreted that different MEG patterns of the eOA at $20-45 \mathrm{~Hz}$ between speech and nonspeech sounds indicated the existence of a fast mechanism for representing and identifying speech sounds in the human auditory cortex.

Schizophrenia is characterized by positive symptoms (e.g., hallucination, delusion), negative symptoms (e.g., affective flattening, anhedonia), and disorganization of thought perhaps re- 
lated to abnormal language processing. Several studies investigated the eOA at $\sim 40 \mathrm{~Hz}$ to auditory "nonspeech" sounds in schizophrenia (Haig et al., 2000; Gallinat et al., 2004). However, it would be important to investigate MEG patterns of the early eOA to speech sounds in schizophrenia, because auditory verbal hallucinations are often shown in schizophrenia and abnormalities in language processing have been proposed as one of the bases for schizophrenia (Nestor et al., 2001).

The present study was designed to test the hypothesis that the schizophrenics will show abnormal early neural oscillatory activity to speech sounds, as measured by the $\mathrm{eOA}$ at $20-45 \mathrm{~Hz}$ within $150 \mathrm{~ms}$ after stimulation, and that this abnormal eOA will be associated with the severity of auditory hallucinations.

\section{Materials and Methods}

Subjects. Twenty patients (15 males) and 23 normal control subjects ( 13 males) with normal hearing (age 18-57, all right handed) participated in this study. After a complete description of the study, all participants signed an informed consent form according to the regulations of the Ethics Commission of the Graduate School of Medical Sciences, Kyushu University. Demographic data for all subjects are presented in Table 1. The exclusion criteria were as follows: (1) neurologic illness or major head trauma that would result in abnormal electroencephalography (EEG); (2) electroconvulsive therapy; (3) alcohol or drug dependence; (4) alcohol or drug abuse within the past five years; or (5) a verbal intelligence quotient $<75$. Normal controls were screened using the Structured Clinical Interview (SCID), nonpatient edition. No normal controls had an Axis I psychiatric disorder in themselves or their first-degree relatives.

All patients were recruited from Kyushu University Hospital and were diagnosed to have schizophrenia based on the SCID for the Diagnostic and Statistical Manual of Mental Disorders, edition IV, and medical records. All patients were receiving neuroleptic medication, with a mean daily dose equivalent to $550 \pm 325 \mathrm{mg}$ of chlorpromazine [typical neuroleptics ( 1 of the 20 patients), atypical (9), or both (10)]. The Scale for the Assessment of Positive Symptoms (SAPS) and the Scale for the Assessment of Negative Symptoms (SANS) were administered to patients. Socioeconomic status (SES) of subjects and parental SES were measured using the Hollingshead two-factor index.

Stimulation and procedures. During the experiment, all subjects were in the lateral recumbent position on a bed in a magnetically shielded room at Kyushu University Hospital. Auditory evoked responses in both hemispheres were recorded alternately (left and right hemispheres counterbalanced between subjects). The subjects were instructed to keep their eyes open and not to sleep. A $2000 \mathrm{~Hz}$ steady-state pure tone was used as the auditory nonspeech stimulus, and the Japanese vowel sound /a/ was used as the auditory speech stimulus. The speech stimulus was the voice spoken by an actor who was a native Japanese speaker. The frequencies for the formants (F) of the vowel /a/ were as follows: F0 $=140 \mathrm{~Hz}, \mathrm{~F} 1=$ $760 \mathrm{~Hz}, \mathrm{~F} 2=1250 \mathrm{~Hz}, \mathrm{~F} 3=2750 \mathrm{~Hz}$, and F4 $=3600 \mathrm{~Hz}$. Both stimuli were edited with $200 \mathrm{~ms}$ duration (rise/fall time, $10 \mathrm{~ms}$ ). At the earpiece, the intensity of each stimulus was the $60 \mathrm{~dB}$ sound pressure level.

Stimuli were delivered to the ear contralateral to the hemisphere being recorded through a $2.3 \mathrm{~m}$ plastic tube with a plastic insert earpiece at the tip. Both stimuli were presented in a pseudorandom order until the sum of the numbers of each stimulus was up to 440 . The interstimulus intervals were between 1 and $2 \mathrm{~s}$. The subjects were instructed to ignore the auditory stimuli.

Data acquisition and data analysis. A 37-channel biomagnetometer was used. The sampling frequency was $4167 \mathrm{~Hz}$. The whole sweep time was $300 \mathrm{~ms}$ (from -50 to $250 \mathrm{~ms}$ after stimulation). Epochs with signal variations exceeding $4.0 \mathrm{pT}$ were excluded. To extract eOA at $20-45 \mathrm{~Hz}$, all of the epochs and the averaged epochs for each condition were applied to the continuous wavelet transform (CWT). CWT was performed using the Matlab Wavelet Toolbox. The complex Morlet wavelet was used as the mother wavelet: $W(t)=\left(\pi f_{\mathrm{b}}\right)^{-0.5} \times \exp \left(-t^{2} / f_{\mathrm{b}}{ }^{2}\right) \times \exp \left(2 i \pi f_{\mathrm{c}} t\right) . f_{\mathrm{b}}$ is a bandwidth parameter, and $f_{\mathrm{c}}$ is a wavelet center frequency parameter. In this analysis, $f_{\mathrm{b}}$ and $f_{\mathrm{c}}$ were chosen to be 1 . We used scales from 167 to 52 in the CWT, corresponding approximately to $20-45 \mathrm{~Hz}$. We used the squared modulus of the result of CWT on the averaged epochs as the eOA power. The square-root transform was applied to the eOA power for normalization, and the mean eOA power of the prestimulus baseline (from -50 to $0 \mathrm{~ms}$ ) was subtracted from the poststimulus $\mathrm{eOA}$ power for each frequency for baseline correction (Kiebel et al., 2005). The eOA power maps of the 10 channels with the largest power within $20-45 \mathrm{~Hz}$ and $0-150 \mathrm{~ms}$ were averaged to insure a reasonably high signal-to-noise ratio (Palva et al., 2002) (see Fig. $1 A$ ). We also calculated eOA phaselocking using the following formula: phase-locking $(t)=1 / N \times|\Sigma z(t)|$, where $N$ is the number of epochs and $z(t)$ refers to the continuous phase of a single trial. Note that $z$ is a complex value on the unit circle, i.e., $z=$ $\exp (j \theta)$, where $\theta$ is the phase of an oscillation and $j$ is the imaginary unit (Tallon-Baudry et al., 1996). We applied baseline correction to and averaged the eOA phase-locking across 10 channels in the same way and channels in the analyses of the eOA power. The eOA power and phaselocking peak latency was defined as the latency with the largest power and phase-locking averaged across $20-45 \mathrm{~Hz}$ within $0-150 \mathrm{~ms}$. To investigate the eOA power change over time, we divided the eOA power maps at $20-45 \mathrm{~Hz}$ into three periods: $0-50 \mathrm{~ms}, 50-100 \mathrm{~ms}$, and $100-150 \mathrm{~ms}$, and averaged the eOA power across $20-45 \mathrm{~Hz}$ in each period (hereinafter called mean eOA power).

The positive wave elicited by auditory stimulation at around $50 \mathrm{~ms}$ has been defined as the P50, and the negative wave elicited at around $100 \mathrm{~ms}$ has been defined as the N100. P50m and N100m are the magnetic counterparts of P50 and N100, respectively. We also investigated the peak amplitudes and latencies of the $\mathrm{P} 50 \mathrm{~m}$ and the $\mathrm{N} 100 \mathrm{~m}$ in the filtered (1-20 Hz) conventional evoked responses. For each component, the peak root mean square (RMS) was computed with the following formula: $\operatorname{RMS}(t)=\left[\Sigma x(i, t)^{2} / n\right]^{0.5}$, where $x(i, t)$ is the magnetic field for each channel $(i=1-10)$ at time $t$ and $\Sigma x(i, t)^{2} / 10$ is the square mean of the magnetic field of the same 10 channels used in the analysis of the eOA power. The P50m latency was defined as the latency with the largest RMS between 20 and $70 \mathrm{~ms}$. The N100m was also defined as between 80 and $120 \mathrm{~ms}$. We also evaluated the lateralization index (LI) of the mean eOA power, the eOA power and phase-locking peak latency for each subject as 
$(\mathrm{L}-\mathrm{R}) /(|\mathrm{L}|+|\mathrm{R}|)$, with $\mathrm{L}$ and $\mathrm{R}$ being the values in the left and right hemisphere (Kircher et al., 2004).

Statistical analysis. The mean eOA powers were submitted to a repeated-measures ANOVA (rmANOVA) with group as a betweensubjects factor, and stimulus (speech or nonspeech), hemisphere (left or right), and period (three periods) as within-subjects factors. For the eOA power peak latency, phase-locking peak latency, the $\mathrm{P} 50 \mathrm{~m}$, and the $\mathrm{N} 100 \mathrm{~m}, \mathrm{rmANOVAs}$ were performed with group as a between-subjects factor, and stimulus and hemisphere as within-subjects factors. LI of these indices were submitted to the same rmANOVA without a hemisphere factor.

The associations between the clinical symptoms and the mean eOA powers to speech sounds in patients were investigated, where the increased severity of auditory hallucinations was predicted to be significantly associated with reduced eOA power. Correlations between auditory hallucinations and the mean eOA powers were evaluated using Spearman's rho.

\section{Results}

\section{Demographics}

There were no significant group differences in the age, handedness, or parental SES. Patients had significantly lower SES than normal controls $\left(t_{(41)}=-5.91 ; p<0.001\right)$, consistent with reduced functioning because of the disorder (Table 1). In addition, there was no significant correlation between the medication dose and eOA $(-0.22 \leq$ rho $\leq 0.43 ; 0.06 \leq p \leq 0.99)$.

eOA power peak latency and eOA phase-locking peak latency We show the topography of the magnetic field and of the spatial derivative at the eOA power peak latency and at the N100m in Figure $1 B$. Spatial derivative was computed with the following formula: spatial derivative $=|\nabla B(u, v)|=\left((\partial B / \partial u)^{2}+(\partial B /\right.$ $\left.\partial v)^{2}\right)^{0.5}$, where $B(u, v)$ is measured axial magnetic field, and $u$ and $v$ are local coordinates (Bastiaansen and Knösche, 2000). Figure $1 C$ shows the grand average eOA power maps. The eOA power maps show clear oscillatory activity at $20-45 \mathrm{~Hz}$ in both groups for each condition. Figure $1 D$ shows the eOA power and phaselocking waveforms and the auditory evoked responses for each condition. The eOA power and phase-locking peak latencies and LI are shown in Figure 2.

In terms of the eOA power peak latency, an rmANOVA demonstrated a significant main effect of group $\left(F_{(1,41)}=7.1 ; p=\right.$ $0.01)$ and hemisphere by stimulus by group $\left(F_{(1,41)}=15.5 ; p<\right.$ $0.001)$ interaction, with no other significant main effects or interactions $\left(0.1 \leq F_{(1,41)} \leq 2.9 ; 0.09 \leq p \leq 0.74\right)$. To delineate this hemisphere by stimulus by group interaction, a follow-up rmANOVA was performed for each stimulus.

For speech sounds, the rmANOVA showed a significant main effect of group $\left(F_{(1,41)}=4.8 ; p=0.04\right)$ and hemisphere by group interaction $\left(F_{(1,41)}=14.0 ; p=0.001\right)$, with no main effect of hemisphere $\left(F_{(1,41)}=0.6 ; p=0.43\right)$. To delineate this hemisphere by group interaction, the differences of the eOA power peak latency between the groups were investigated using $t$ tests for each hemisphere. In the left hemisphere, patients showed significantly delayed eOA power peak latencies $\left(t_{(41)}=-3.9 ; p=0.001\right)$. In the right hemisphere, there was no significant difference in the eOA power peak latencies between the groups $\left(t_{(41)}=0.3 ; p=0.78\right)$. The eOA power peak latencies were (in ms, mean \pm SD) $85.5 \pm$ 29.0 for patients and $57.3 \pm 15.8$ for normal controls in the left hemisphere, and $67.1 \pm 24.1$ and $69.2 \pm 24.5$ in the right hemisphere. For nonspeech sounds, the rmANOVA showed a significant hemisphere by group interaction $\left(F_{(1,41)}=5.0 ; p=0.03\right)$, with no main effects $\left(0.3 \leq F_{(1,41)} \leq 2.9 ; 0.10 \leq p \leq 0.60\right)$. In the left hemisphere, there was no significant difference in the eOA power peak latency between groups $\left(t_{(41)}=0.003 ; p=1.0\right)$. In the right hemisphere, patients showed significantly delayed eOA latencies $\left(t_{(41)}=-2.9 ; p=0.005\right)$. The peak latencies were $71.0 \pm$ 16.8 for patients and $71.0 \pm 20.1$ for normal controls in the left hemisphere, and $80.0 \pm 19.7$ and $65.5 \pm 12.3$ in the right hemisphere, respectively. For LI of the eOA power peak latency, an rmANOVA demonstrated a significant stimulus by group $\left(F_{(1,41)}\right.$ $=14.9 ; p<0.001)$ interaction, with no main effects $\left(0.8 \leq F_{(1,41)}\right.$ $\leq 2.6 ; 0.11 \leq p \leq 0.39$ ). To delineate this stimulus by group interaction, the differences of LI between the groups were investigated using t tests for each stimulus. For both stimuli, patients showed the opposite hemispheric patterns to normal controls $\left(t_{(41)}=-3.5, p=0.001\right.$ for speech sounds; $t_{(41)}=2.2, p=0.04$ for nonspeech sounds) (Fig. 2). For the eOA phase-locking peak latency, the statistical results were the same as the results of the eOA power peak latency (Fig. 2, supplemental information and Fig. 1, available at www.jneurosci.org as supplemental material).

In summary, patients showed a delayed neural oscillatory activity to speech sounds in the left hemisphere and to nonspeech sounds in the right hemisphere, while also showing the opposite hemispheric patterns of eOA.

\section{Mean eOA power}

The mean eOA power and LI are shown in Figure 2. An rmANOVA demonstrated significant main effects of period $\left(F_{(2,82)}=69.7 ; p<0.001\right)$ and stimulus $\left(F_{(1,41)}=9.1 ; p=0.004\right)$, and significant hemisphere by stimulus $\left(F_{(1,41)}=4.9 ; p=0.032\right)$, period by stimulus $\left(F_{(2,82)}=22.0 ; p<0.001\right)$, and period by hemisphere by stimulus by group $\left(F_{(2,82)}=3.6 ; p=0.038\right)$ interactions, with no other significant main effects or interactions $\left(0.09 \leq F_{(1,41)} \leq 1.2 ; 0.7 \leq F_{(2,82)} \leq 2.7 ; 0.08 \leq p \leq 0.77\right)$. To delineate this period by hemisphere by stimulus by group interaction, the hemisphere factor was decomposed first.

In the left hemisphere, the rmANOVA showed a significant main effect of period $\left(F_{(2,82)}=40.9 ; p<0.001\right)$, and significant period by group $\left(F_{(2,82)}=3.7 ; p=0.038\right)$, period by stimulus $\left(F_{(2,82)}=5.7 ; p=0.008\right)$, and period by stimulus by group $\left(F_{(2,82)}\right.$ $=3.4 ; p=0.049)$ interactions, with no other significant main effects or interactions $\left(0.01 \leq F_{(1,41)} \leq 0.6 ; 0.46 \leq p \leq 0.94\right)$. To disentangle this period by stimulus by group interaction, follow-up rmANOVA was performed for each stimulus.

For speech sounds, the rmANOVA showed a significant main effect of period $\left(F_{(2,82)}=16.4 ; p<0.001\right)$ and period by group $\left(F_{(2,82)}=5.7 ; p=0.009\right)$ interaction, with no main effect of group $\left(F_{(1,41)}=0.02 ; p=0.9\right)$. To delineate this period by group interaction, the differences of the mean eOA power between the groups were investigated using $t$ tests for each period. In the $0-50$ ms period, normal controls showed a significantly larger mean eOA power $\left(t_{(41)}=2.2 ; p=0.03\right)$ than patients. In the $50-100 \mathrm{~ms}$ period, there was no significant difference in the mean eOA power between the groups $\left(t_{(41)}=0.2 ; p=0.88\right)$. In the $100-150$ ms period, patients showed a significantly larger mean eOA power $\left(t_{(41)}=-2.5 ; p=0.02\right)$ than normal controls. For nonspeech sounds, the rmANOVA showed a significant main effect of period $\left(F_{(2,82)}=50.6 ; p<0.001\right)$, with no other significant main effect or interaction $\left(F_{(1,41)}<0.001 ; F_{(2,82)}=0.7 ; 0.5 \leq p \leq\right.$ $1.0)$. The mean eOA powers in the left hemisphere to speech sounds were (in $\mathrm{fT} / \mathrm{cm}$ ) $22.6 \pm 16.7$ for patients and $36.2 \pm 22.0$ for normal controls in the $0-50 \mathrm{~ms}$ period, $43.2 \pm 31.2$ and $44.5 \pm 23.2$ in the $50-100 \mathrm{~ms}$ period, and $28.1 \pm 21.2$ and $15.1 \pm$ 11.2 in the $100-150 \mathrm{~ms}$ period, and to nonspeech sounds were $23.2 \pm 14.4$ for patients and $27.6 \pm 14.7$ for normal controls, 

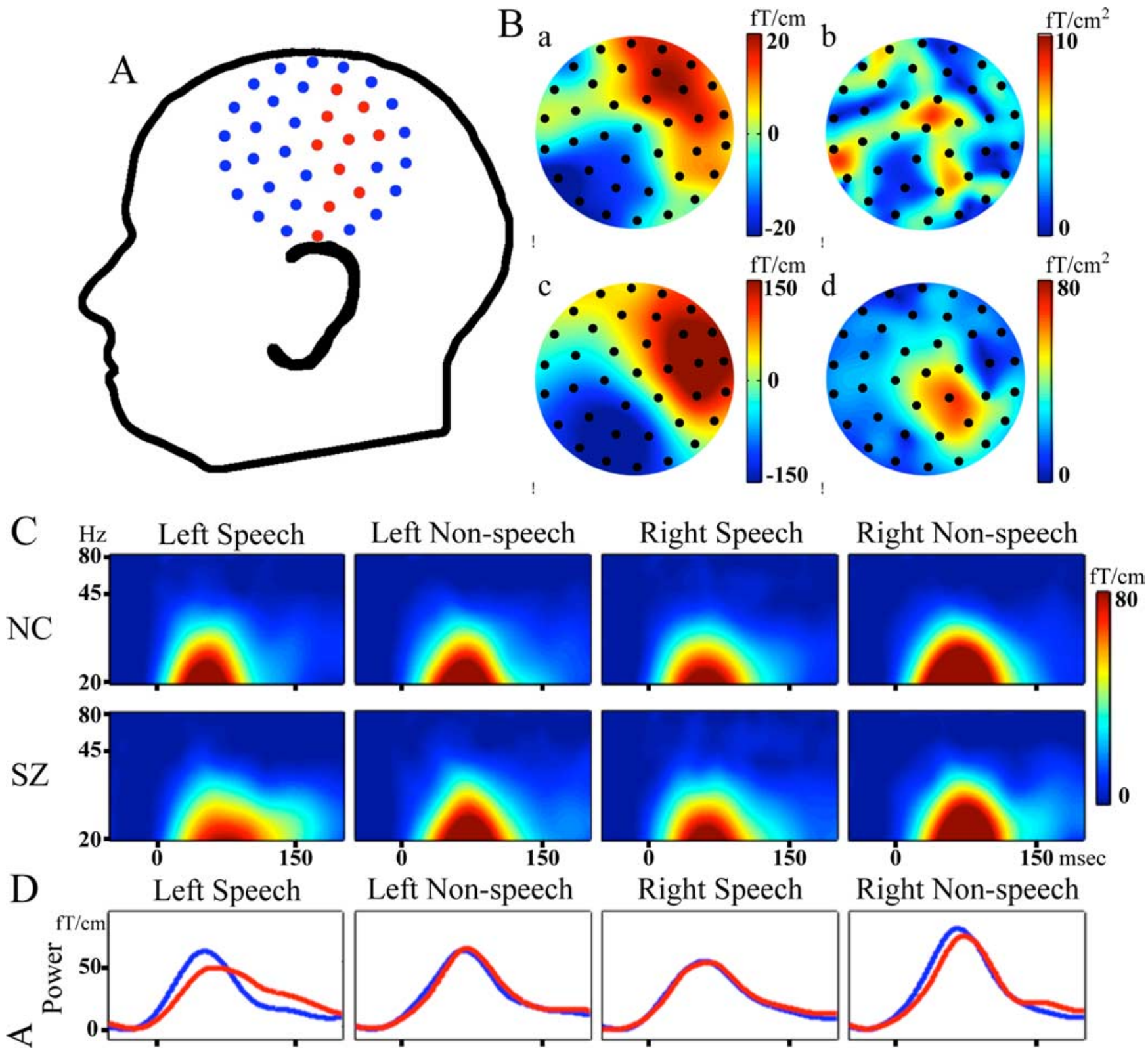

SZ

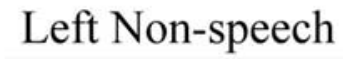

Right Speech
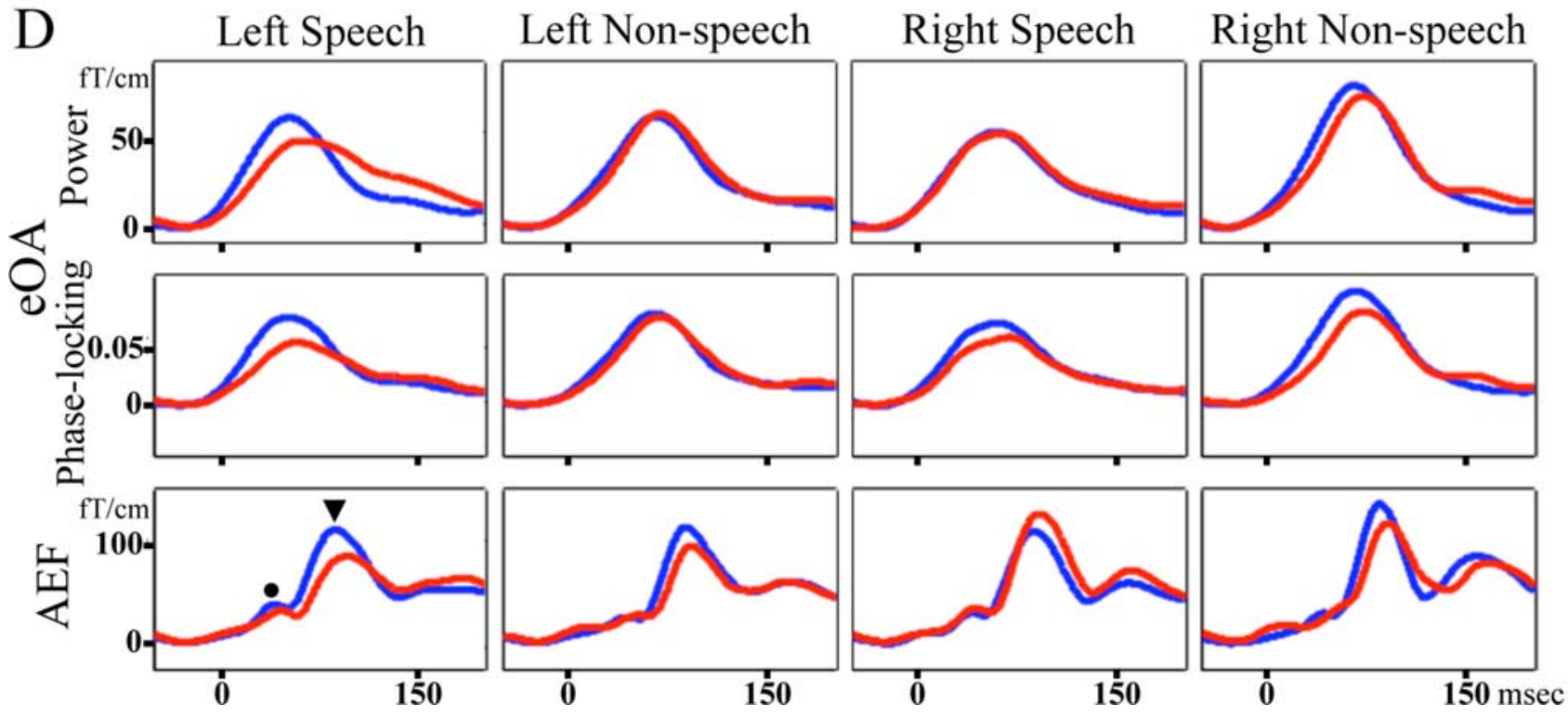

Figure 1. $\quad \boldsymbol{A}$ and $\boldsymbol{B}$ are based on the data to speech sounds in the left hemisphere of one subject. $\boldsymbol{A}$, The layout of the measured channels. Red circles indicate the channels used for the analyses. $\boldsymbol{B} \boldsymbol{a}$, The topography of magnetic field (MF) at the eOA power peak latency. $\boldsymbol{B} \boldsymbol{b}$, Spatial derivative of $\boldsymbol{B} \boldsymbol{a}$. $\boldsymbol{B} \boldsymbol{c}$, MF of the N100m. $\boldsymbol{B} \boldsymbol{d}$, Spatial derivative of $\boldsymbol{B} \boldsymbol{c}$. Color scales signify the MF and spatial derivative. $C$, Grand average $\mathrm{e} 0 \mathrm{~A}$ power maps to speech and nonspeech sounds in the both hemispheres. Color scale signifies e OA power. D, Top, Grand average e $0 \mathrm{~A}$ power waveforms. Middle, Grand average e $0 \mathrm{~A}$ phase-locking waveforms. Bottom, Grand average auditory evoked responses. The black circle indicates the P50m, and the black triangle indicates the $\mathrm{N} 100 \mathrm{~m}$. The waveforms of patients are shown in red and those of normal controls are shown in blue. NC, Normal controls; SZ, patients with schizophrenia; AEF, auditory evoked magnetic field. 

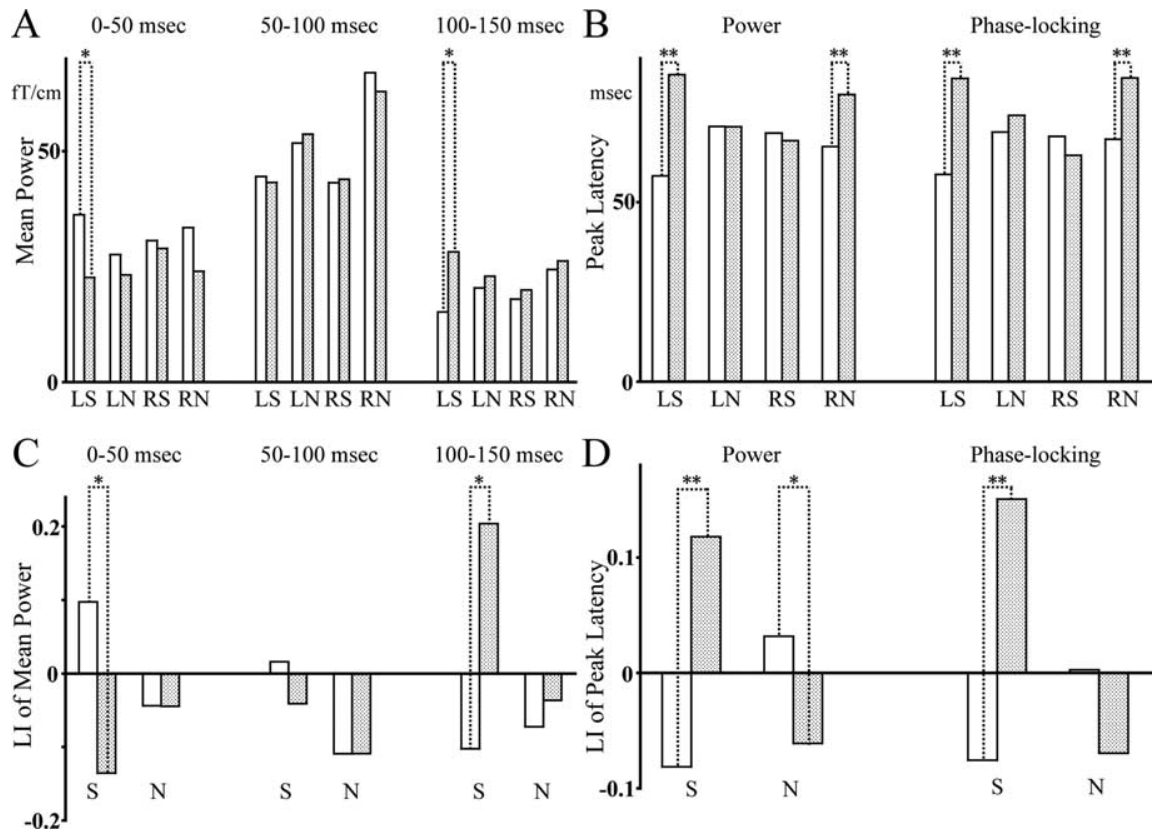

Figure 2. $\quad \boldsymbol{A}$, The mean eOA power. $\boldsymbol{B}$, The eOA power and phase-locking peak latency. $\boldsymbol{C}, \mathrm{Ll}$ of $\boldsymbol{A} . \boldsymbol{D}, \mathrm{Ll}$ of $\boldsymbol{B}$. $\mathrm{L}$ and $R$ are the left and right hemisphere, and $S$ and $N$ are speech and nonspeech sounds, respectively. The data of patients are shown as dotted bars and those of normal controls are shown as plain bars. ${ }^{* *} p<0.01 ;{ }^{*} p<0.05$.

$53.7 \pm 29.2$ and $51.7 \pm 25.6$, and $22.9 \pm 13.1$ and $20.4 \pm 11.2$, respectively.

In the right hemisphere, the rmANOVA demonstrated a significant main effects of period $\left(F_{(2,82)}=55.2 ; p<0.001\right)$ and stimulus $\left(F_{(1,41)}=11.3 ; p=0.002\right)$, and a significant period by stimulus $\left(F_{(2,82)}=20.1 ; p<0.001\right)$ interaction, with no other significant main effects or interactions $\left(0.2 \leq F_{(1,41)} \leq 0.6 ; 0.6 \leq\right.$ $\left.F_{(2,82)} \leq 0.7 ; 0.43 \leq p \leq 0.67\right)$. The mean eOA powers in the right hemisphere to speech sounds were (in $\mathrm{fT} / \mathrm{cm}$ ) $29.0 \pm 17.9$ for patients and $30.6 \pm 24.5$ for normal controls in the $0-50 \mathrm{~ms}$, $43.9 \pm 22.3$ and $43.2 \pm 22.7$ in the $50-100 \mathrm{~ms}$, and $19.9 \pm 15.6$ and $18.0 \pm 10.2$ in the $100-150 \mathrm{~ms}$, and to nonspeech sounds were $23.9 \pm 12.9$ for patients and $33.4 \pm 23.6$ for normal controls, $62.9 \pm 27.8$ and $67.0 \pm 33.8$, and $26.2 \pm 16.3$ and $24.4 \pm$ 13.0 , respectively.

For the LI of the mean eOA power, an rmANOVA demonstrated a significant period by group $\left(F_{(2,82)}=3.6 ; p=0.04\right)$ and period by stimulus by group $\left(F_{(2,82)}=5.4 ; p=0.01\right)$ interactions, with no other significant main effects or interactions $(0.005 \leq$ $\left.F_{(1,41)} \leq 2.2 ; 0.58 \leq F_{(2,82)} \leq 0.61 ; 0.15 \leq p \leq 0.95\right)$. To delineate this period by stimulus by group interaction, a follow-up rmANOVA was performed for each stimulus. For speech sounds, an rmANOVA demonstrated a significant period by group $\left(F_{(2,82)}=7.9 ; p=0.003\right)$ interaction, with no main effects $\left(F_{(1,41)}\right.$ $\left.=0.003 ; F_{(2,82)}=0.6 ; 0.49 \leq p \leq 0.96\right)$. To delineate this period by group this interaction, the differences in the LI between the groups were investigated using $t$ tests for each period. Patients showed the opposite hemispheric patterns to normal controls in the $0-50 \mathrm{~ms}$ and $100-150 \mathrm{~ms}$ periods, with no difference in the $50-100 \mathrm{~ms}$ period $\left(0-50 \mathrm{~ms}\right.$ period, $t_{(41)}=2.1, p=0.04 ; 50-100$ ms period, $t_{(41)}=0.5, p=0.62 ; 100-150 \mathrm{~ms}$ period, $t_{(41)}=-2.2$, $p=0.03$ ) (Fig. 2). We also used the other baseline (from -100 to $-50 \mathrm{~ms}$ ) for the analyses to confirm the results, and the statistical results were the same using both baselines.

In summary, patients showed a significantly smaller neural oscillatory activity in the $0-50 \mathrm{~ms}$ period and a significantly larger activity in the $100-150 \mathrm{~ms}$ in the left hemisphere to speech sounds specifically, and showed opposite hemispheric patterns of eOA to speech sounds in the two periods. The results of eOA in $45-80 \mathrm{~Hz}$ are presented in the supplemental material (available at www.jneurosci.org).

\section{P50m and N100m}

For the P50m amplitude, an rmANOVA demonstrated a significant main effect of stimulus $\left(F_{(1,41)}=8.9 ; p=0.005\right)$, with no other significant main effects or interactions $\left(0.07 \leq F_{(1,41)} \leq 2.4 ; 0.13 \leq p \leq\right.$ 0.79). For the P50m latency, there were no significant main effects or interactions $\left(0.001 \leq F_{(1,41)} \leq 0.9 ; 0.35 \leq p \leq 0.97\right)$.

For the N100m amplitude, an rmANOVA demonstrated a significant main effect of hemisphere $\left(F_{(1,41)}=5.3\right.$; $p=0.03)$, with no other significant main effects or interactions $\left(0.2 \leq F_{(1,41)} \leq 3.6\right.$; $0.07 \leq p \leq 0.69)$. For the N100m latency, there were a significant main effect of stimulus $\left(F_{(1,41)}=11.1 ; p=0.002\right)$, but no other significant main effects or interactions $\left(0.004 \leq F_{(1,41)} \leq 3.2 ; 0.08 \leq p \leq\right.$ $0.95)$. These results indicated that there were no significant group differences in the P50m and the $\mathrm{N} 100 \mathrm{~m}$ of the conventional evoked responses.

\section{Correlations between eOA power and auditory hallucination}

A significant negative correlation was observed between the mean eOA power to speech sounds in the 100-150 ms period in the left hemisphere and the auditory hallucination scores in SAPS ( rho = $-0.51 ; p=0.023$ ), but no significant correlations between the eOA power peak latency to speech sounds in the left hemisphere, those to nonspeech sounds in the right hemisphere, nor any LI of these indices $(-0.41 \leq$ rho $\leq 0.19 ; 0.07 \leq p \leq 1.0)$ (Fig. 3$)$.

\section{Discussion}

The major findings of this study were as follows: (1) patients showed a delayed neural oscillatory activity to speech sounds in the left hemisphere and to nonspeech sounds in the right hemisphere; (2) in the $0-50 \mathrm{~ms}$ period, patients showed significantly reduced eOA power to speech sounds in the left hemisphere; (3) in the 100-150 ms period, patients showed significantly larger eOA power to speech sounds in the left hemisphere; (4) patients showed the opposite hemispheric patterns in eOA peak latency to both sounds and the opposite hemispheric patterns in eOA to speech sounds; (5) the eOA power in 100-150 ms period to speech sounds in the left hemisphere was associated with auditory hallucinations.

\section{Abnormal eOA to speech sounds in schizophrenia}

Näätänen et al. (1997) reported the existence of a cortical language-specific automatic detection system indexed by the mismatch negativity. This system is formed within the first year of life (Cheour et al., 1998) and it has been hypothesized to underlie the activation of the speech-specialized network in the left hemisphere. Based on these studies, Palva et al. (2002) stated that the different MEG patterns of the eOA at $20-45 \mathrm{~Hz}$ to speech and nonspeech sounds in humans might reflect the activation of the 
left-hemispheric language-specialized network. The results of the current study suggest that patients with schizophrenia may have deficits in a fast mechanism for identifying speech sounds, related to an impaired left hemispheric language-specific neural system. Moreover, in the present study, the analyses of the lateralization index revealed the pattern of hemispheric lateralization to be the opposite in patients. A reversal in normal lateralization may therefore be closely related to the pathology of schizophrenia.

For the eOA-symptom correlation, the increased severity of auditory hallucinations was associated with smaller eOA to speech sounds in the left hemisphere in the 100-150 ms period. The results of the current study indicate that this abnormal neural oscillatory activity to speech sounds may underlie the generation of auditory hallucinations in schizophrenia; however, this finding warrants confirmation in a larger sample.

\section{Generation of the eOA at $20-45 \mathrm{~Hz}$}

Previous in vitro studies have suggested that beta 2 oscillations $(20-30 \mathrm{~Hz})$ are different from gamma oscillations (30-45 $\mathrm{Hz}$ ) in terms of generation. For gamma oscillations, Cunningham et al. (2004) reported that the fast rhythmic bursting neurons in layer II/III play a crucial role in the generation of gamma oscillations. GABAergic neurons have been reported to play a crucial role in the primary generation of gamma oscillations and their local synchronization (Traub et al., 2004). In addition, direct electronic coupling through gap junctions between inhibitory neurons also contributes to the synchronization of the gamma oscillations (Traub et al., 2001). For beta oscillations, in vitro study by Roopun et al. (2006) reported that beta2 oscillations occurred in layer V pyramidal cells. Moreover, this study indicated that beta2 oscillations are involved in gap junctional coupling and independent of chemical synaptic transmission. The present study suggests that schizophrenia may be characterized by a left hemisphereselective impaired neural circuitry for speech detection, and this functional deficit, at the cellular level, may be associated with a reduction of GABA interneurons and/or an abnormality in gap junctions.

\section{Previous studies on eOA in schizophrenia}

To our knowledge, this is the first study to demonstrate abnormal eOA to speech sounds in schizophrenia. Kwon et al. (1999) reported reduced $40 \mathrm{~Hz}$ steady-state eOA and showed delayed phase synchronization/desynchronization to the click train in schizophrenia. MEG offers high spatial resolution to detect neuronal activity to auditory stimuli (Onitsuka et al., 2000) and, thus, MEG is suited to evaluate the hemispheric differences in eOA. Because the $40 \mathrm{~Hz}$ entrained EEG response to auditory stimuli is largest at the middle frontal electrode sites and unfit to detect hemispheric differences, it is the novel finding of the current study that patients showed a delayed neural oscillatory activity to speech sounds in the left hemisphere and to nonspeech sounds in the right hemisphere. It will be important to clarify the associa- tion between the delayed eOA latency of the present study and the delayed phase synchronization/desynchronization to the click train in the same samples.

As noted above, there is a difference in the generation between beta 2 and gamma oscillations. Therefore, the findings in eOA studies for schizophrenia should be carefully interpreted, because some of these studies have investigated high-frequency oscillations including both beta and gamma band oscillations (Blumenfeld and Clementz, 2001).

\section{Conclusion}

These results indicated that patients showed different characteristics of eOA compared with normal controls, probably related to deficits in a fast mechanism for identifying speech sounds, and the abnormal neural circuit function in speech recognition may be an underlying pathophysiology of schizophrenia. Moreover, the present study suggested that schizophrenia might be characterized by an opposite pattern of hemispheric lateralization in auditory evoked oscillations.

\section{References}

Bastiaansen MC, Knösche TR (2000) Tangential derivative mapping of axial MEG applied to event-related desynchronization research. Clin Neurophysiol 111:1300-1305.

Belin P, Zatorre RJ, Lafaille P, Ahad P, Pike B (2000) Voice-selective areas in human auditory cortex. Nature 403:309-312.

Blumenfeld LD, Clementz BA (2001) Response to the first stimulus determines reduced auditory evoked response suppression in schizophrenia: single trials analysis using MEG. Clin Neurophysiol 112:1650-1659.

Cheour M, Ceponiene R, Lehtokoski A, Luuk A, Allik J, Alho K, Näätänen R (1998) Development of language-specific phoneme representations in the infant brain. Nat Neurosci 5:351-353.

Cunningham MO, Whittington MA, Bibbig A, Roopun A, LeBeau FE, Vogt A, Monyer H, Buhl EH, Traub RD (2004) A role for fast rhythmic burst- 
ing neurons in cortical gamma oscillations in vitro. Proc Natl Acad USA 101:7152-7157.

Gallinat J, Winterer G, Herrmann CS, Senkowski D (2004) Reduced oscillatory gamma-band responses in unmedicated schizophrenic patients indicate impaired frontal network processing. Clin Neurophysiol 115:1863-1874.

Haig AR, Gordon E, Pascalis VD, Meares RA, Bahramali H, Harris A (2000) Gamma activity in schizophrenia: evidence of impaired network binding? Clin Neurophysiol 111:1461-1468.

Herrmann CS, Munk MH, Engel AK (2004) Cognitive functions of gammaband activity: memory match and utilization. Trends Cogn Sci 8:347-355.

Hong LE, Summerfelt A, McMahon R, Adami H, Francis G, Elliott A, Buchanan RW, Thaker GK (2004) Evoked gamma band synchronization and the liability for schizophrenia. Schizophr Res 70:293-302.

Kiebel SJ, Tallon-Baudry C, Friston KJ (2005) Parametric analysis of oscillatory activity as measured with EEG/MEG. Hum Brain Mapp 26:170-177.

Kircher TT, Rapp A, Grodd W, Buchkremer G, Weiskopf N, Lutzenberger W, Ackermann H, Mathiak K (2004) Mismatch negativity responses in schizophrenia: a combined fMRI and whole-head MEG study. Am J Psychiatry 161:294-304.

Kwon JS, O'Donnel BF, Wallenstein GV, Greene RW, Hirayasu Y, Nestor PG, Hasselmo ME, Potts GF, Shenton ME, McCarley RW (1999) Gamma frequency-range abnormalities to auditory stimulation in schizophrenia. Arch Gen Psychiatry 56:1001-1005.

Näätänen R, Lehtokoski A, Lennes M, Cheour M, Huotilainen M, Iivonen A, Vainio M, Alku P, Ilmoniemi RJ, Luuk A, Allik J, Sinkkonen J, Alho K (1997) Language-specific phoneme representations revealed by electric and magnetic brain responses. Nature 385:432-434.

Nestor PG, Han SD, Niznikiewicz M, Salisbury DF, Spencer KM, Shenton ME, McCarley RW (2001) Semantic disturbance in schizophrenia and its relationship to the cognitive neuroscience of attention. Biol Psychol $57: 23-46$.

Onitsuka T, Ninomiya H, Sato E, Yamamoto T, Tashiro N (2000) The effect of interstimulus intervals and between-block rests on the auditory evoked potential and magnetic field: is the auditory P50 in humans an overlapping potential? Clin Neurophysiol 111:237-245.

Palva S, Palva JM, Shtyrov Y, Kujala T, Ilmoniemi RJ, Kaila K, Näätänen R (2002) Distinct gamma-band evoked responses to speech and nonspeech sounds in humans. J Neurosci 22:RC211(1-5).

Roopun AK, Middleton SJ, Cunningham MO, LeBeau FE, Bibbig A, Whittington MA, Traub RD (2006) A beta2-frequency (20-30 Hz) oscillation in non synaptic networks of somatosensory cortex. Proc Natl Acad USA 103:15646-15650.

Spencer KM, Nestor PG, Niznikiewicz MA, Salisbury DF, Shenton ME, McCarley RW (2003) Abnormal neural synchrony in schizophrenia. J Neurosci 23:7407-7411.

Spencer KM, Nestor PG., Perlmutter R, Niznikiewicz MA, Klump MC, Frumin M, Shenton ME, McCarley RW (2004) Neural synchrony indexes disordered perception and cognition in schizophrenia. Proc Natl Acad USA 101:17288-17293.

Tallon-Baudry C, Bertrand O, Delpuech C, Pernier J (1996) Stimulus specificity of phase-locked and non-phase-locked $40 \mathrm{~Hz}$ visual responses in human. J Neurosci 16:4240-4249.

Traub RD, Koppell N, Bibbig A, Buhl EH, LeBeau FE, Whittington MA (2001) Gap junctions between interneuron dendrites can enhance synchrony of gamma oscillations in distributed networks. J Neurosci 21:9478-9486.

Traub RD, Bibbig A, LeBeau FE, Buhl EH, Whittington MA (2004) Cellular mechanisms of neuronal population oscillation in the hippocampus in vitro. Annu Rev Neurosci 27:247-278.

Uhlhaas PJ, Singer W (2006) Neural synchrony in brain disorders: relevance for cognitive dysfunctions and pathophysiology. Neuron 52:155-168.

Uhlhaas PJ, Linden DE, Singer W, Haenschel C, Lindner M, Maurer K, Rodriguez E (2006) Dysfunctional long-range coordination of neural activity during Gestalt perception in schizophrenia. J Neurosci 26:8168-8175. 